\title{
GreenLight Laser photoselective vapo-enucleation of the prostate with front-firing emission versus plasmakinetic resection of the prostate for benign prostate hyperplasia
}

\author{
Xiangming Cheng ${ }^{1,2 \#}$, Zikai Qiü ${ }^{2 \#}$, Jie Dong ${ }^{2 \#}$, Guanghua Liu ${ }^{2}$, Yi Xie ${ }^{2}$, Weifeng Xu ${ }^{2}$, Zhigang Ji ${ }^{2}$ \\ ${ }^{1}$ Department of Urology, Affiliated Hospital of Nanjing University of Chinese Medicine, Jiangsu Province Hospital of Traditional Chinese Medicine, \\ Nanjing 210029, China; ${ }^{2}$ Department of Urology, Peking Union Medical College Hospital, Chinese Academy of Medical Science, Beijing 100730, \\ China \\ Contributions: (I) Conception and design: X Cheng, W Xu, Z Ji; (II) Administrative support: Z Ji; (III) Provision of study materials or patients: J \\ Dong, G Liu; (IV) Collection and assembly of data: Z Qiu, Y Xie; (V) Data analysis and interpretation: X Cheng, Z Qiu; (VI) Manuscript writing: All \\ authors; (VII) Final approval of manuscript: All authors. \\ "These authors contributed equally to this work. \\ Correspondence to: Prof. Weifeng Xu; Prof. Zhigang Ji. Department of Urology, Peking Union Medical College Hospital, Chinese Academy of \\ Medical Science, Beijing 100730, China. Email: pumchxwf@yeah.net; jzg1129@yeah.net.
}

Background: Although the conventional, monopolar transurethral resection of the prostate (TURP) has proven to be an effective and relatively safe treatment for patients with benign prostatic hyperplasia $(\mathrm{BPH})$, many new endoscopic technologies have been introduced to treat BPH. With the development of laser, there are several alternative transurethral procedures embracing laser therapies. Herein, this study sought to explore the efficacy, safety and follow-up of GreenLight laser photoselective vapo-enucleation of the prostate (PVEP) with front-firing emission compared with plasmakinetic resection of the prostate (PKRP) used to surgically manage BPH.

Methods: Data from patients who underwent either GreenLight laser PVEP or PKRP were retrospectively collected from March 2013 to May 2018. Perioperative data from both groups were compared.

Results: Totally, 43 and 45 patients were included in the PVEP and PKRP groups, respectively. No significant difference was observed in excision efficiency ratio (resected prostate weight/operation time) between the two groups $(\mathrm{P}=0.372)$. The efficiency ratio of the first $20 \mathrm{PVEP}$ procedures $(0.36 \pm 0.09 \mathrm{~g} / \mathrm{min})$ was significantly lower than that of the second $23 \mathrm{PVEP}$ procedures $(0.45 \pm 0.18 \mathrm{~g} / \mathrm{min})(\mathrm{P}=0.042)$. The PVEP group experienced a shorter duration of catheterization, postoperative hospital stay and irrigation time than the PKRP group $(\mathrm{P}<0.001, \mathrm{P}=0.001$ and $\mathrm{P}<0.001$, respectively). There was no statistically significant difference between the two groups $(\mathrm{P}=0.937)$ in terms of overall postoperative complications. Three months after surgery, the international prostate symptoms (IPSS) score, quality of life (QOL) score, postvoid residual (PVR) volume and maximum urinary flow rate $\left(\mathrm{Q}_{\max }\right)$ were decreased in both groups $(\mathrm{P}<0.001$ for all $)$ and were comparable between both groups $(\mathrm{P}=0.635,0.662,0.671$ and 0.924 , respectively).

Conclusions: GreenLight laser PVEP with front-firing emission was safe and effective modality in treating patients with BPH with short-term follow-up. PVEP was associated with shorter catheterization and postoperative hospital stay time compared with PKRP.

Keywords: GreenLight laser; vapo-enucleation; plasmakinetic resection of the prostate (PKRP); benign prostate hyperplasia

Submitted Sep 08, 2019. Accepted for publication Jan 17, 2020.

doi: $10.21037 /$ tau.2020.02.04

View this article at: http://dx.doi.org/10.21037/tau.2020.02.04 


\section{Introduction}

Lower urinary tract symptoms (LUTS) secondary to benign prostatic hyperplasia (BPH) are highly prevalent in men older than 50 years of age. Surgery is indicated in cases involving complications or moderately to severely bothersome symptoms that cannot be adequately addressed via drug treatments (1). For a long time, transurethral resection of the prostate (TURP) has been regarded as the standard surgical treatment for small to mediumsized prostates (2). However, numerous alternatives have challenged the efficacy and safety of conventional TURP because of its considerable complications (3). One of the alternatives that has been studied is bipolar transurethral resection of the prostate (B-TURP), which uses saline irrigation instead of mannitol solutions to decrease the risk of TUR syndrome and improve the rate of immediate complications such as hemoglobin drop, transfusion and immediate reoperation (2).

With the development of laser technology, there have been several alternative transurethral procedures developed embracing laser therapies. Compared to B-TURP, holmium-laser enucleation of the prostate (HoLEP) not only has a larger amount of prostate tissue retrieved but is also associated with an increased reduction of bladder irrigation, catheter time and hospital stay (4). However, despite these advantages, HoLEP is still limited to expert teams at high volume centers because of its steep learning curve (5). Among energy sources, GreenLight $532 \mathrm{~nm}$ laser photoselective vaporization of the prostate (PVP) has emerged as an appealing treatment modality for treating small to medium glands because of satisfying hemostatic effects and a relatively short learning curve $(6,7)$ but has been associated with high long-term reoperation rates for larger glands and the absence of postoperative pathologic tissues, even in the $180-W$ Xcelerated Performance System (XPS) era $(8,9)$. A GreenLight laser with adoption of the enucleation principle has recently been advocated as a new endoscopic enucleation procedure in treating BPH (10). A randomized, controlled study indicated comparable perioperative outcomes between the HoLEP and photoselective vapo-enucleation of the prostate (PVEP) with a side-firing GreenLight laser, but more patients in the PVEP group required hospital stay for more than one night due to hematuria (7). For hemostasis in the enucleation procedure, front-firing might be easier than side-firing. However, few studies have reported the efficacy and safety of PVEP with front-firing GreenLight lasers. Furthermore, the perioperative outcomes of GreenLight front-firing laser PVEP compared with B-TURP are still unknown.

In our study, GreenLight laser PVEP with front-firing emission was explored and employed for BPH treatment. In addition, its efficacy, safety and follow-up were compared with plasmakinetic resection of the prostate (PKRP), which is a type of B-TURP.

\section{Methods}

\section{Patients}

After receiving institutional review board approval, data from patients who were referred for LUTS secondary to BPH and who underwent surgery were gathered from March 2013 to May 2018. Patients who underwent PKRP or PVEP with front-firing GreenLight laser performed by a single surgeon were included regardless of the prostate measurement. Patients with prostate cancer diagnosed preoperatively, active bladders, neurological disorders or pelvic radiotherapy were excluded from the retrospective study. Patients with prostate-specific antigen $(\mathrm{PSA})>4 \mathrm{ng} / \mathrm{mL}$ or an abnormal finding on digital rectal examination underwent prostate magnetic resonance imaging (MRI) and ultrasound-guided prostate biopsy prior to surgery. The patients were provided with informed consent to undergo PKRP or the Greenlight laser PVEP.

\section{Invention}

\section{PKRP}

All operations were performed by one surgeon with rich clinical experience in PKRP procedures with a 26 Fr Karl Storz continuous flow resectoscope and a reusable plasmaloop electrode. PKRP was operated with a cutting power of 120-160 watts and a coagulating power of 60-80 watts. Physiologic saline $(0.9 \% \mathrm{NaCl})$ was used as irrigation fluid. The patients were placed in the lithotomy position. The procedure began with resection of the median lobe. A longitudinal groove deepened into the surgical capsule was first made at the 6 o'clock position from the bladder neck to the proximal margin of the verumontanum. After the median lobe was resected, the bilateral lobe was resected in sequence to completely remove the prostate adenoma. At the end of each operation, continuous isotonic irrigation was performed on all patients by inserting a $22 \mathrm{Fr}$ urethral catheter. The catheter was usually removed 2-3 days after the operation or when the patient's hematuria ceased. 

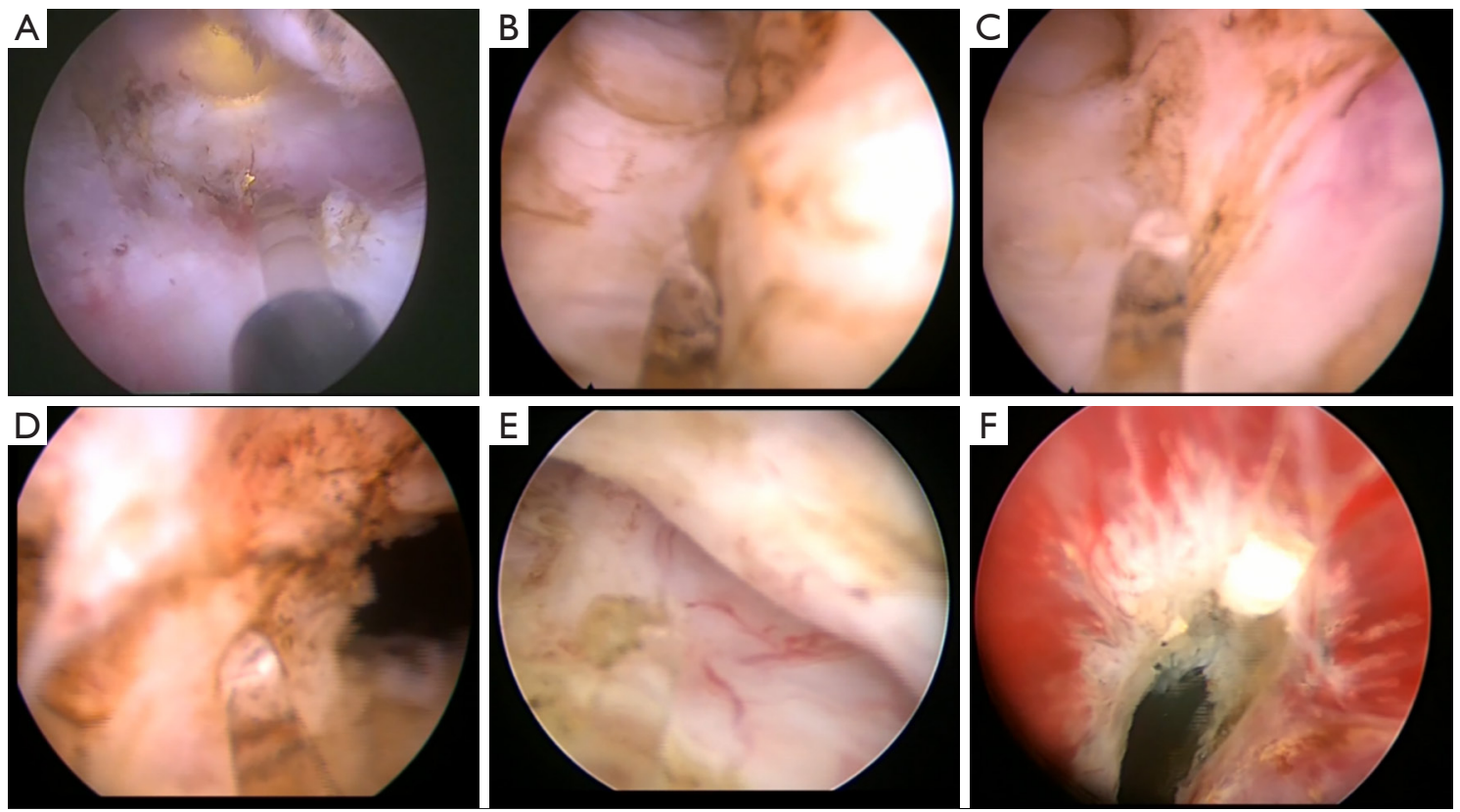

Figure 1 The vital steps of Greenlight laser PVEP. (A) The surgical capsule found at the 5-o'clock position near verumontanum; (B) the incision between the bilateral lobe and median lobe from the bladder neck to the operating plane marked by the surgical capsule; (C) the incision was performed in the front of the verumontanum; (D) the median lobe peeled off the surgical capsule; (E) the left lobe enucleated along or a little bit above the surgical capsule between 5 and 2 o'clock; (F) bladder-neck incision made at the 12 o'clock position through adenomatous tissue to the prostatic capsule.

\section{Greenlight laser PVEP}

The equipment used was a laser equipment $180 \mathrm{~W}$ (XPS) Greenlight laser treatment system (made in China) with front-firing fiber, a continuous flow 26 Fr Storz resectoscope and a video system. The patients were placed in the lithotomy position and under video control. The laser energy was set at 60-160 watts for enucleation and $30 \mathrm{~W}$ for coagulation. Normal saline was used as the irrigant throughout the procedure. The steps of the technique are described below. (I) Exposing the posterior surgical capsule at the apex. PVEP began at the apex with an incision at the 5-o'clock and 7-o'clock positions about $1 \mathrm{~cm}$ away from verumontanum. The incision was deepened with a distal extension until the surgical capsule was reached (Figure 1A). (II) Enucleation of the median lobe. Marked by the surgical capsule, two longitudinal grooves deep into the capsule were incised between the bilateral lobe and the median lobe from the bladder neck to the operating plane at 5 and 7 o'clock (Figure 1B). Then, the median lobe was dissected with the bilateral lobe. Another incision was performed at the front of the verumontanum (Figure 1C). The median lobe was then peeled off the surgical capsule toward the neck of the bladder (Figure 1D). Attention was paid to protecting the muscle fibers of the neck of the bladder. (III) Enucleation of the bilateral lobe. Enucleation of the left lateral lobe started with a semicircular incision of the apical tissue between 5 and 2 o'clock along or a little bit above the surgical capsule when the capsule was not clear (Figure 1E). Then, a bladder neck incision was made at the 12 o'clock position through adenomatous tissue to the prostatic capsule (Figure $1 F$ ). This incision was then connected to the 2 o'clock incision with blunt dissection of the sheath. This was repeated in a similar manner for enucleation of the right lateral lobe. (IV) Vaporization of residual glands, careful hemostasis and tissue morcellation. The laser power was set at 120-160 watts to vaporize residual glands. After hemostasis, the mechanical tissue morcellator was activated through the nephroscope to rapidly remove the floating prostate lobes. A standard $22 \mathrm{Fr}$ urethral catheter was placed in the patient at the end of the procedure.

\section{Data collection}

The perioperative data were retrospectively reviewed 
and recorded. The baseline characteristics included the American Society of Anaesthesiologists (ASA) score, body mass index (BMI), serum prostate-specific antigen (PSA) level, prostate volume assessed by transrectal ultrasound, the international prostate symptom (IPSS) score, quality of life (QOL), postvoid residual (PVR) volume, maximum urinary flow rate $\left(\mathrm{Q}_{\max }\right)$, usage of 5-alpha-reductase inhibitors (5ARIs) and/or alpha blockers, and usage of antiplatelet or anticoagulation therapy and indwelling catheters. In addition, the following perioperative data were collected: operative time, efficiency ratio (defined as resected prostate weight/operation time), resected prostate volume, reduction rate of prostate volume, catheterization time, postoperative hospitalization time, postoperative irrigation time, histopathology and complications. General postoperative complications were graded according to the Clavien-Dindo classification (11). The changes in subjective (IPSS and QOL) and objective (PVR and $\mathrm{Q}_{\max }$ ) urinary parameters were assessed at 3-month follow-up.

\section{Statistical analysis}

Data are presented as the mean \pm standard deviation (SD) or the medians (range) for quantitative variables and frequencies combined with percentages for categorical variables. The results were compared between treatment groups using the Chi-square test or Fisher's exact test for categorical variables and the Student's $t$-test, the paired $t$-test or Mann-Whitney $\mathrm{U}$ test for quantitative variables. All statistical analyses were performed using SPSS 21.0 software (SPSS Inc., Chicago, IL, USA). A P value $<0.05$ was considered statistically significant.

\section{Results}

After reviewing the medical history of patients in our institution, a total of 88 patients were included in the final analysis. Greenlight laser PVEP with front-firing emission was performed in 43 cases, and PKRP procedures were applied in 45 cases. The patients' baseline characteristics are listed in Table 1. The ASA score of patients in the PVEP group was significantly higher than that in the PKRP group $(\mathrm{P}=0.018)$, while there was no statistically significant difference observed in age between both groups $(\mathrm{P}=0.7)$. Other clinical features, including prostate volume, BMI, proportion of cases with indwelling catheters and PSA level, were comparable between the PVEP and PKRP groups $(\mathrm{P}=0.2,0.6,0.7$ and 0.7 , respectively). For symptoms, since the percentage of patients receiving 5ARIs and alpha blockers were similar in both groups $(\mathrm{P}=0.3$ and 0.8 , respectively), there was no significant difference in IPSS score and QOL score ( $\mathrm{P}=0.6$ and 0.9 , respectively). The patients with bladder stones were treated with holmium laser for free before the procedure.

All Greenlight laser PVEP procedures were successfully performed. No significant difference was observed in terms of efficiency ratio (resected prostate weight/operation time) between the two groups $(\mathrm{P}=0.4)$. The excision efficiency ratio of the first 20 PVEP procedures $(0.36 \pm 0.09 \mathrm{~g} / \mathrm{min})$ was significantly lower than that of the second 23 PVEP procedures $(0.45 \pm 0.18 \mathrm{~g} / \mathrm{min})(\mathrm{P}=0.042)$. The weight of the resected prostate was significantly higher, and the operation time was longer, in the PVEP group than in the PKRP group $(\mathrm{P}<0.001$ for both measures). No case was converted to PKRP for hemostasis and residual tissue removal in the PVEP group. In addition, patients in the PVEP group experienced a shorter duration of catheterization, postoperative hospital stay and irrigation time than those in the PKRP group $(\mathrm{P}<0.001, \mathrm{P}=0.001$ and $\mathrm{P}<0.001$, respectively).

Regarding overall postoperative complications ( $<30$ days), there was no statistically significant difference between the two groups $(\mathrm{P}=0.9)$, which is shown in Table 2. The rates of urinary incontinence (UI) and capsular perforations in the PVEP group were higher than those in the PKRP group, although no statistical difference was observed $(\mathrm{P}=0.6)$. Adult diapers were applied for patients with urinary incontinence. The extended duration of catheterization until irrigated fluid was completely clear could be beneficial to patients with capsular perforations. The PKRP had 1 patient with a Clavien-Dindo grade of $3 \mathrm{~b}$ receiving re-cystoscopy for hemostasis, and the PVEP group had 1 patient with an internal urethrotomy for urethral stricture.

The short-term clinical outcomes of these two procedures were evaluated at 3 months after surgery with collection of the following data: IPSS score, QOL score and PVR volume measured by ultrasound, which is shown in Table 3. The IPSS score, QOL score and PVR volume were decreased after 3 months in both groups $(\mathrm{P}<0.001$ for all). The test of $\mathrm{Q}_{\max }$ was not administered for all patients with $\mathrm{BPH}$ : only 10 patients in the PKRP group and 9 patients in the PVEP group received the test preoperatively and postoperatively. $\mathrm{Q}_{\max }$ was improved after surgery in both groups $(\mathrm{P}<0.001$ for all). In addition, the IPSS score, QOL score, PVR volume and $\mathrm{Q}_{\max }$ were comparable between 
Table 1 Perioperative characteristics of patients

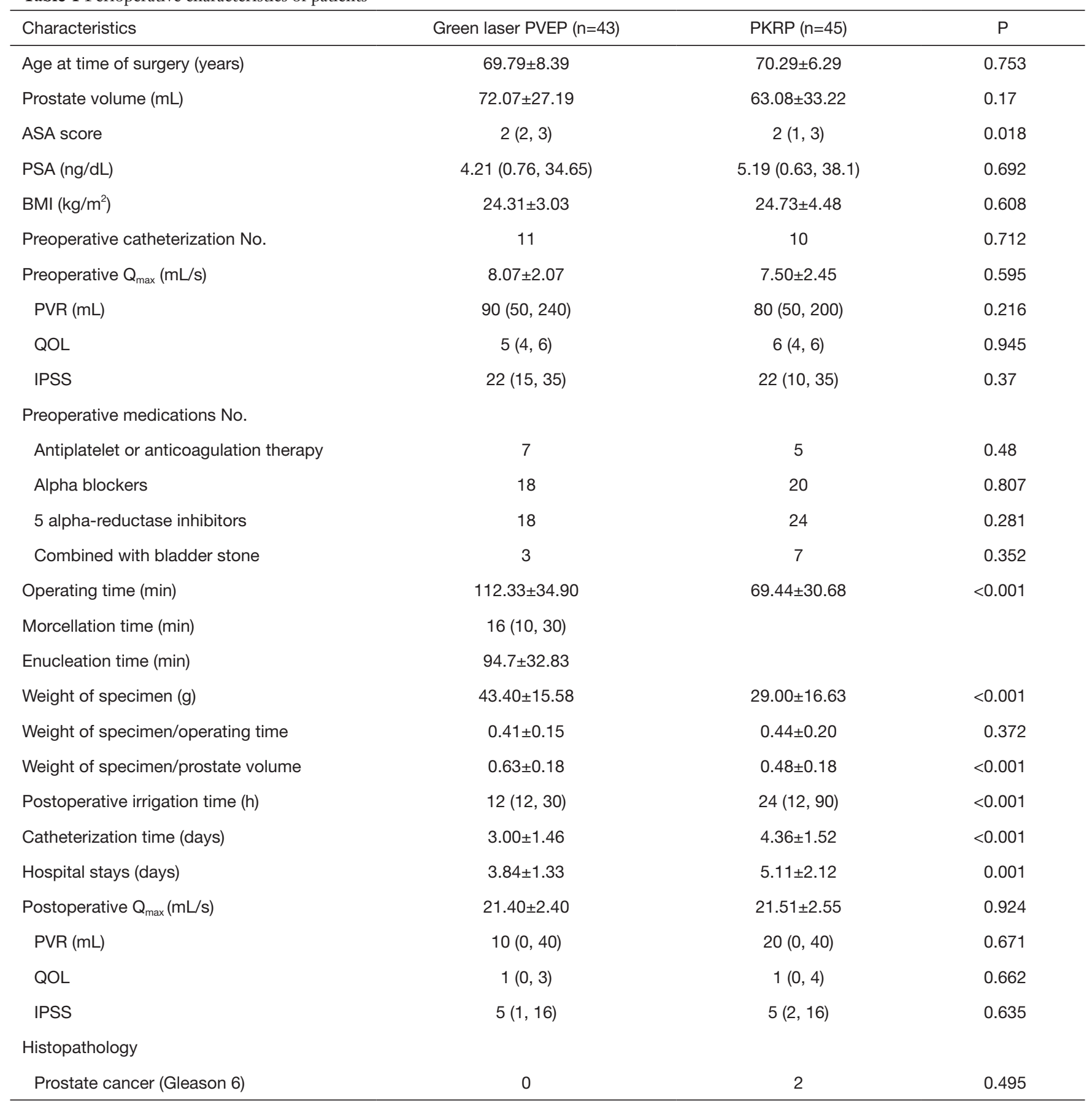

both groups 3 months after surgery $(\mathrm{P}=0.6,0.7,0.7$ and 0.9 , respectively).

\section{Discussion}

Although conventional, monopolar TURP has proven to be an effective and relatively safe treatment for patients with BPH, many new endoscopic technologies have been introduced to treat BPH. PKRP, one of a group of resection technologies called B-TURP, is widely used and has been proposed to replace monopolar TURP, as it shows better perioperative data and fewer immediate complications than 
Table 2 Postoperative complications

\begin{tabular}{|c|c|c|c|c|c|}
\hline Clavien-Dindo grade & Complications & Green laser PVEP & PKRP & $\mathrm{P}$ & Management \\
\hline Grade 1 & Capsular perforations & 1 & 0 & 0.489 & Overtime of catheterization \\
\hline Grade 2 & Gross hematuria requiring transfusion & 0 & 0 & - & - \\
\hline Grade 3a & Ureteral orifice injury & 0 & 0 & - & - \\
\hline Grade 3b & $\begin{array}{l}\text { Gross hematuria with a lot of blood clot } \\
\text { in bladder }\end{array}$ & 0 & 1 & 1.000 & $\begin{array}{l}\text { Re-cystoscopy and } \\
\text { hemostasis }\end{array}$ \\
\hline All complications & - & 3 & 2 & 0.937 & - \\
\hline
\end{tabular}

Table 3 Short-term functional outcomes

\begin{tabular}{|c|c|c|c|c|c|c|}
\hline Outcomes & \multicolumn{3}{|c|}{ Green laser PVEP } & \multicolumn{3}{|c|}{ PKRP } \\
\hline PVR (mL) & $90(50,240)$ & $10(0,40)$ & $<0.001$ & $80(50,200)$ & $20(0,40)$ & $<0.001$ \\
\hline QOL & $5(4,6)$ & $1(0,3)$ & $<0.001$ & $6(4,6)$ & $1(0,4)$ & $<0.001$ \\
\hline IPSS & $22(15,35)$ & $5(1,16)$ & $<0.001$ & $22(10,35)$ & $5(2,16)$ & $<0.001$ \\
\hline
\end{tabular}

monopolar TURP as well as comparable short-term and long-term efficacy outcomes $(2,12)$. Endoscopic enucleation of the prostate (EEP), another promising modality of prostatic tissue ablation, has been reported to be operated by various types of technologies such as holmium laser, thulium laser, electroenucleation and so on (13). Until now, several studies have reported that the clinical outcomes of using a Greenlight laser with side-firing emission employed for enucleation are noninferior to those of HoLEP and lower peri-operative morbidity rate than those of open prostatectomy $(7,14,15)$. Unfortunately, side-firing fibers for enucleation mainly relies on mechanical, blunt, "energy-free" dissection (15) and is not convenient for sharp dissection and hemostasis when separating tissues from the surgical capsule. This may hinder the adoption of the Greenlight laser in enucleation, as the difficulty of the enucleation procedure is not only in the identification of the plane between the surgical capsule and adenoma without severe bleeding in the operative site but also in maintaining one plane throughout the procedure using both blunt and sharp dissection (16), especially for newcomers performing more sharp dissections in EEP. In addition, the emission window of the side-firing fiber is prone to damage via heat reflection and tissue debris, which severely impairs transmission of the laser energy (17). Therefore, a frontfiring laser is essential for the utilization of GreenLight laser PVEP. To our knowledge, this study is the first to report the use of a front-firing GreenLight laser for prostate enucleation. Additionally, the efficacy, safety and follow-up of PVEP with front-firing laser were compared with PKRP in the present study.

The well-known advantage of the GreenLight laser is the appealing hemostatic effects since the wavelength can be selectively absorbed by hemoglobin (6). In our study, complications associated with gross hematuria were not observed in the PVEP group. However, in two previous studies on GreenLight PVEP with side-firing lasers, PVEP had more bleeding requiring auxiliary TURP $(11 \%$ and $24.5 \%$ ) for hemostasis compared with HoLEP, although the hemostatic effect of the holmium laser was not better than that of GreenLight laser $(7,15)$. Another study on the $180-W$ XPS system of PVP reported that bleeding resulting in impaired visualization was the most common intraoperative complication (10\%), and a TURP loop had to be resorted 
in most of these patients (7.7\%) (18). These results may demonstrate that the side-firing laser was not convenient and effective for hemostasis during enucleation. The pressure of arterial bleeding was likely high, and a front-firing laser fiber tip was able to be pressed directly onto bleeding points to stop the blood flow, but it was difficult for a side-firing laser. At the same time, the bladder irrigation time was shorter in the PVEP group than in the PKRP group, and the bladder irrigation color in the PVEP group appeared clearer than that in the PKRP group in our study. The reason may lie in the good hemostatic effect of the Greenlight laser and the fact that gland removal along the envelope layer during PVEP is beneficial to reducing wound bleeding.

Capsular perforation, another complication in EEP due to enucleation along the surgical capsule, has been documented in between $0.3 \%$ to $10 \%$ of cases involving the HoLEP procedure (19). For the PVEP procedure with a side-firing laser, it was reported that the occurrence rate was $5.6-11.6 \%(7,15,20)$. Potential risk factors included the size of the prostate, and newer surgeons' experiences with longer operative times and suboptimal observation. The rate of capsular perforation in PVEP in our study was only $2.3 \%$, which occurred in the first 20 procedures. The surgeon could maintain the capsule well depending on optimal observation resulting from the appealing hemostatic effect of the GreenLight laser.

Postoperative UI is one of the main complications affecting the quality of life of patients after enucleation of the prostate. Previous studies reported that the occurrence of postoperative UI after GreenLight laser PVEP was $3.4-25 \%(7,14,20)$ whereas the rate was $4.7 \%(2 / 43)$ in the PVEP group of our study. These rates were similar to the rates reported in the literature with HoLEP (1.444\%) (20), and the rates in HoLEP was reported to be improved with increasing surgical experience of handling the prostate apex and sphincter as well as controlling the extent of resectoscope movement (21). In our study, with increasing experience, we found that saving some of the urethral mucosa at the prostate apex was helpful for preventing sphincter injury and avoiding UI. Furthermore, a low energy of approximately 60 watts was applied when handling adenoma at the prostate apex to avoid urethral thermal injury leading to urethral stricture. The incidence of urethral stricture was $2.3 \%(1 / 43)$ in our study. High energy might be the reason why the incidence of urethral stricture reached $20 \%$ in the PVP procedure (20).

The outcomes of GreenLight front-firing laser PVEP were recorded. In the current study, a high power of 120 watts for the vaporization effect and a low power of 30 watts for the coagulation effect were employed for residual prostate removal and hemostasis, respectively, after the PVEP procedure with a power of $60-80$ watts. Despite the effect of vaporization, the resected weight of the prostate in the PVEP group was larger than that in the PKRP group, which was similar to perioperative results from prospective randomized studies comparing HoLEP with B-TURP $(4,22)$. The catheterization and hospitalization times in the present study were consistent with those observed in prior comparative studies of PKRP/EEP with different energy sources $(4,23)$ and were significantly shorter for the PVEP group than for the PKRP group. The operative time of the PVEP procedures was longer than that of PKRP in our study. However, the operative time of PVEP with the GreenLight laser was believed to be shorter as the number of performances increased since excision efficiency ratio of the second 23 procedures was higher than that of the first 20 PVEP procedures by a single surgeon. After the 32 th case, complete enucleation could be achieved within 120 min without postoperative complications or stress urinary incontinence at 3 months. These advantages make the technique safe for EEP and easy for surgeons to learn. Thus, patients with higher ASA scores tended to be selected to receive PVEP rather than PKRP after we finished 20 PVEP procedures. Therefore, the ASA score in the PVEP group was higher than in the PKRP group.

One of the limitations of the present study is the shortterm follow-up. However, considering that this report focuses on describing the technical aspects of PVEP and presenting our initial experience with this novel technique, short-term follow-up seems to be acceptable. In addition, the influence of PVEP on sexual function and the learning curve for surgeons remain to be explored. Last but not least, small sample size leading to the absence of significant result in the complication rates.

\section{Conclusions}

GreenLight laser PVEP with front-firing emission was safe and effective modality in treating patients with $\mathrm{BPH}$ with short-term follow-up. PVEP was associated with shorter catheterization and postoperative hospital stay time compared with PKRP. Further study with expanded sample size is needed to ensure long-term durability of the outcome. 


\section{Acknowledgments}

The authors thank Dr. Jingmin Zhou and Dr. Dong Wang for their constructive suggestions during the preparation of the article.

Funding: None.

\section{Footnote}

Conflicts of Interest: All authors have completed the ICMJE uniform disclosure form (available at http://dx.doi. org/10.21037/tau.2020.02.04). The authors have no conflicts of interest to declare.

Ethical Statement: The authors are accountable for all aspects of the work in ensuring that questions related to the accuracy or integrity of any part of the work are appropriately investigated and resolved. The Institutional Review Board of Peking Union Medical College Hospital approve our review and patients were consented for the publication.

Open Access Statement: This is an Open Access article distributed in accordance with the Creative Commons Attribution-NonCommercial-NoDerivs 4.0 International License (CC BY-NC-ND 4.0), which permits the noncommercial replication and distribution of the article with the strict proviso that no changes or edits are made and the original work is properly cited (including links to both the formal publication through the relevant DOI and the license). See: https://creativecommons.org/licenses/by-nc$\mathrm{nd} / 4.0 \%$.

\section{References}

1. Oelke M, Bachmann A, Descazeaud A, et al. EAU guidelines on the treatment and follow-up of nonneurogenic male lower urinary tract symptoms including benign prostatic obstruction. Eur Urol 2013;64:118-40.

2. Cornu JN, Ahyai S, Bachmann A, et al. A Systematic Review and Meta-analysis of Functional Outcomes and Complications Following Transurethral Procedures for Lower Urinary Tract Symptoms Resulting from Benign Prostatic Obstruction: An Update. Eur Urol 2015;67:1066-96.

3. Rassweiler J, Teber D, Kuntz R, et al. Complications of transurethral resection of the prostate (TURP)--incidence, management, and prevention. Eur Urol 2006;50:969-79; discussion 980.

4. Chen YB, Chen Q, Wang Z, et al.A prospective, randomized clinical trial comparing plasmakinetic resection of the prostate with holmium laser enucleation of the prostate based on a 2-year followup. J Urol 2013;189:217-22.

5. Robert G, Cornu JN, Fourmarier M, et al. Multicentre prospective evaluation of the learning curve of holmium laser enucleation of the prostate (HoLEP). BJU Int 2016;117:495-9.

6. Te AE. The Next Generation in Laser Treatments and the Role of the GreenLight High-Performance System Laser. Rev Urol 2006;8 Suppl 3:S24-30.

7. Elshal AM, Elkoushy MA, El-Nahas AR, et al. GreenLight laser (XPS) photoselective vapo-enucleation versus holmium laser enucleation of the prostate for the treatment of symptomatic benign prostatic hyperplasia: a randomized controlled study. J Urol 2015;193:927-34.

8. Meskawi M, Hueber PA, Valdivieso R, et al. Multicenter international experience of $532 \mathrm{~nm}$-laser photovaporization with Greenlight XPS in men with large prostates (prostate volume $>100 \mathrm{cc}$ ). World J Urol 2017;35:1603-9.

9. Hueber PA, Ben-Zvi T, Liberman D, et al. Mid term outcomes of initial 250 case experience with GreenLight $120 \mathrm{~W}$-HPS photoselective vaporization prostatectomy for benign prostatic hyperplasia: comparison of prostate volumes $<60 \mathrm{cc}, 60 \mathrm{cc}-100 \mathrm{cc}$ and $>100 \mathrm{cc}$. Can J Urol 2012;19:6450-8.

10. Gomez Sancha F, Rivera VC, Georgiev G, et al. Common trend: move to enucleation-Is there a case for GreenLight enucleation? Development and description of the technique. World J Urol 2015;33:539-47.

11. Clavien PA, Barkun J, de Oliveira ML, et al. The ClavienDindo classification of surgical complications: five-year experience. Ann Surg 2009;250:187-96.

12. Liu Z, Li YW, Wu WR, et al. Long-term Clinical Efficacy and Safety Profile of Transurethral Resection of Prostate Versus Plasmakinetic Resection of the Prostate for Benign Prostatic Hyperplasia. Urology 2017;103:198-203.

13. Enikeev D, Glybochko P, Rapoport L, et al. A randomized trial comparing the learning curve of three endoscopic enucleation techniques (HoLEP, ThuFLEP and MEP) for $\mathrm{BPH}$ using mentoring approach - initial results. Urology 2018;121:51-7.

14. Misraï V, Pasquie M, Bordier B, et al. Comparison between open simple prostatectomy and green laser enucleation of the prostate for treating large benign prostatic hyperplasia: 
a single-centre experience. World J Urol 2018;36:793-9.

15. Peyronnet B, Robert G, Comat V, et al. Learning curves and perioperative outcomes after endoscopic enucleation of the prostate: a comparison between GreenLight 532$\mathrm{nm}$ and holmium lasers. World J Urol 2017;35:973-83.

16. Elshal AM, Nabeeh H, Eldemerdash Y, et al. Prospective Assessment of Learning Curve of Holmium Laser Enucleation of the Prostate for Treatment of Benign Prostatic Hyperplasia Using a Multidimensional Approach. J Urol 2017;197:1099-107.

17. Gong YG, Liu RM, Gao R. Photoselective Vaporesection of the Prostate with a Front-firing Lithium Triborate Laser: Surgical Technique and Experience After 215 Procedures. Eur Urol 2015;67:1152-9.

18. Bachmann A, Muir GH, Collins EJ, et al. 180-W XPS GreenLight laser therapy for benign prostate hyperplasia: early safety, efficacy, and perioperative outcome after 201 procedures. Eur Urol 2012;61:600-7.

19. Shah HN, Mahajan AP, Hegde SS, et al. Peri-operative complications of holmium laser enucleation of the

Cite this article as: Cheng X, Qiu Z, Dong J, Liu G, Xie Y, $\mathrm{Xu}$ W, Ji Z. GreenLight Laser photoselective vapo-enucleation of the prostate with front-firing emission versus plasmakinetic resection of the prostate for benign prostate hyperplasia. Transl Androl Urol 2020;9(2):544-552. doi: 10.21037/tau.2020.02.04 prostate: experience in the first 280 patients, and a review of literature. BJU Int 2007;100:94-101.

20. Misrai V, Kerever S, Phe V, et al. Direct Comparison of GreenLight Laser XPS Photoselective Prostate Vaporization and GreenLight Laser En Bloc Enucleation of the Prostate in Enlarged Glands Greater than $80 \mathrm{ml}$ : a Study of 120 Patients. J Urol 2016;195:1027-32.

21. Shigemura K, Yamamichi F, Kitagawa K, et al. Does Surgeon Experience Affect Operative Time, Adverse Events and Continence Outcomes in Holmium Laser Enucleation of the Prostate? A Review of More Than 1,000 Cases. J Urol 2017;198:663-70.

22. Fayad AS, Elsheikh MG, Zakaria T, et al. Holmium Laser Enucleation of the Prostate Versus Bipolar Resection of the Prostate: A Prospective Randomized Study. "Pros and Cons". Urology 2015;86:1037-41.

23. Yang $Z$, Wang $X$, Liu T. Thulium laser enucleation versus plasmakinetic resection of the prostate: a randomized prospective trial with 18-month follow-up. Urology 2013;81:396-400. 